\title{
Simple and Fast Method for Identification and Quantification of Anthocyanidins in Berries by Ultra Performance Liquid Chromatography-Mass Spectrometry
}

\author{
Julianna M. Vagula, ${ }^{a}$ Nayane M. Sinosaki, ${ }^{a}$ Marcos A. S. Ribeiro, ${ }^{b}$ Thiago Magon, ${ }^{a}$ \\ Janksyn Bertozzi, ${ }^{c}$ Eduardo C. Meurer, ${ }^{b}$ Oscar O. Santos Junior ${ }^{a}$ and \\ Jesuí V. Visentainer*,a \\ ${ }^{a}$ Departamento de Química, Universidade Estadual de Maringá, Av. Colombo, \\ 87020-900 Maringá-PR, Brazil \\ ${ }^{b}$ Laboratório FENN de Espectrometria de Massas, Universidade Federal do Paraná, \\ 86900-000 Jandaia do Sul-PR, Brazil \\ ${ }^{c}$ Departamento de Química, Universidade Tecnológica Federal do Paraná, \\ 86036-370 Londrina-PR, Brazil
}

\begin{abstract}
The objective was identifying three different anthocyanidins and to quantify them in six different berries. Results from the conventional method (using extraction with hydrochloric acid) were compared with data from the proposed method (without acid extraction). The following figures of merit were used: linearity, accuracy, linear range, limits of detection and quantification, using an ultra performance liquid chromatography (UPLC) coupled to a triple quadrupole mass spectrometer equipped with an electrospray ionization source. The separation of anthocyanidins can be achieved in a much smaller period of time (1.30 min) and the cyanidin from Morus nigra L., Rubus idaeus L. and Vaccinium myrtillus L. was found in higher amounts in extracts obtained from the proposed method. Proposed mechanisms of mass spectrometry (MS/MS) collisional induced dissociation for pelargonidin, cyanidin and delphinidin were obtained and this is the first time that concentrations of anthocyanidins present in fruits of Solanum americanum Mill. were reported.
\end{abstract}

Keywords: electrospray ionization, positive mode, cyaniding, pelargonidin, delphinidin

\section{Introduction}

In order to perform the basic functions of nutrition, cell renewal and respiration, all plants perform photosynthesis, which involves the primary metabolism, characterized by essential biochemical transformations in the generation of energy, being indispensable the insertion of macronutrients in this metabolism. ${ }^{1,2}$

Some plant species, in addition to primary metabolism, also developed secondary metabolism, such development is dependent on the genetic predisposition of each plant organism. This secondary metabolism generates chemical compounds with diverse biological activities. Such metabolism may be: (i) initiated after a specific stimulation, such as: ultraviolet radiation, temperature change, lack of nutrients and presence of pathogens, or (ii) present in the plant starting from its embryonic stage,

*e-mail: jesuiv@ gmail.com serving as an additional defense system of the vegetal organism. ${ }^{2,3}$

Phenolic compounds are derived from this secondary metabolism, mainly by the shikimic acid route and to a lesser extent by the mevalonic acid pathway, and they are subdivided into other important groups that vary according to the number of phenols present in the molecules. One of these groups is called flavonoids, which is subdivided into 6 other classes, such as flavonone, flavanol, flavonol, flavone, anthocyanidin and isoflavone. ${ }^{4,5}$

Anthocyanidins constitute the most important class of natural pigments after chlorophyll. They are polar, water-soluble molecules, highly unstable to light and storage temperature, and they can be obtained from the metabolism of acetate and chiquimate, derived from the flavylium cation. ${ }^{6}$ These pigments are present in berries, a generic term called for small fruits, with small seeds, which can be eaten whole. Usually, most of the edible berries are highly healthy foods, rich in nutrients 
and antioxidants. The fruits that contain peel and pulp with purple coloration may present high content of anthocyanins (chromogenic substances found in red, blue and purple fruits). Compounds with antioxidant activity are substances that can inhibit the reactions of free radicals, such as reactive species of oxygen, besides also acting as inhibitors of carcinogenesis. ${ }^{2,6}$

In the human organism, anthocyanins have biological functions against free radicals derived from normal human metabolism, this function of minimizing free radicals is related to the prevention of cardiovascular, circulatory, inflammatory and cancerous diseases, among others. Foods rich in phytonutrients included in diet have preventive action regarding the development of cardiovascular diseases. $^{?}$

In order to identify these compounds, different types of assays can be used for the analysis of antioxidant compounds, such as DPPH• (2,2-diphenyl-1-picrylhydrazyl), a direct method, and the ORAC (oxygen radical absorbance capacity), an indirect method. However, it is necessary to develop faster and more sensitive analytical methodologies for determining the concentrations of compounds with antioxidant properties, mainly in fruits, characterized as complex samples to be analyzed. ${ }^{8}$

In this context, high performance liquid chromatography and mass spectrometry are used for this purpose, ${ }^{8}$ for developing a simple and fast method. To reduce or eliminate the use or generation of harmful substances to human health and environment, it was prioritized the development and the implementation of the processes. ${ }^{9,10}$

Therefore, this research was focused on the development and validation of a fast method for the determination of three different types of anthocyanidins using ultra performance liquid chromatography coupled to mass spectrometry (UPLC-MS/MS).

\section{Experimental}

\section{Chemicals and standards}

The analytical standards of delphinidin chloride, cyanidin chloride and pelargonidin chloride, all of purity greater than 95\%, were supplied by Sigma-Aldrich (St. Louis, USA). Formic acid and hydrochloric acid (98\%) were purchased from Millipore-Sigma (Darmstadt, Germany). Methanol (HPLC-grade) was purchased from Panreac (Barcelona, Spain). Ultrapure water was obtained through Milli-Q system (Millipore, Bedford, USA). All samples were passed through a $0.22 \mu \mathrm{m}$ Millipore transfer membrane of polyvinylidene fluoride (Tullagreen, Carringtwonill Co., Ireland).
Plant material

The ripe fruits of Morus nigra L. (black mulberry), Prunus avium L. (cherry), Rubus idaeus L. (raspberry), Solanum americanum Mill. (black nightshade), Vaccinium myrtillus L. (blueberry) and Vitis vinifera L. (grapes) were purchased from the local commerce in

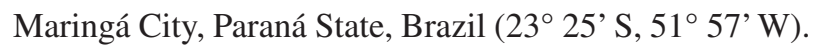
Samples were sanitized, homogenized and immediately applied in the extraction procedures.

\section{Conventional method}

Method validation was carried out through comparison of the results obtained by the proposed method with those obtained by the conventional assay. The employed conventional extraction method was previously described, ${ }^{11}$ in which $15 \mathrm{~g}$ of sample was diluted in acidified methanol $(\mathrm{pH} 3)$ and sonicated on ultrasonic bath (Elmasonic $\mathrm{P}$, Elma $^{\odot}$, Germany) for $20 \mathrm{~min}$, followed by filtration $(0.22$ $\mu \mathrm{m}$ membrane). Sample extracts were immediately injected in the UPLC-MS/MS equipment.

\section{Proposed method}

Fruits were manually macerated, the obtained juices were isolated and filtered in a $0.22 \mu \mathrm{m}$ membrane. The $\mathrm{pH}$ of each extract was measured for comparison, being approximately the same ( $\mathrm{pH} 3)$ due to the acid compounds present in the studied fruits. Samples were immediately injected in the UPLC-MS/MS equipment.

\section{Instrumentation}

The extracts were injected in an UPLC Acquity system coupled to a triple quadrupole mass spectrometer (MS) equipped with an electrospray ionization source (ESI, Waters Zspray ${ }^{\mathrm{TM}}$, Waters, Millford, MA). Mobile phase (A) was composed of ultrapure water acidified with $0.1 \%$ formic acid while mobile phase (B) was composed of methanol acidified with $0.1 \%$ formic acid, both with $\mathrm{pH} 3$. The mass spectrometry determination was performed with an electrospray source in the positive ion mode; capillary voltage: $3 \mathrm{kV}$; cone voltage: $80 \mathrm{~V}$; electrospray tension: $3 \mathrm{kV}$; desolvation gas temperature: $300{ }^{\circ} \mathrm{C}$; desolvation gas flow: $800 \mathrm{~L} \mathrm{~h}^{-1}$; collision gas: set in a pressure of $3.5 \mathrm{mBar}$. Extracts were separated on an Acquity UPLC ${ }^{\circledR}$ Bridged Ethane Hybrid $(\mathrm{BEH}) \mathrm{C} 18$ column $(50 \times 2.1 \mathrm{~mm}, 1.7 \mu \mathrm{m})$. Elution was performed in isocratic mode using $45 \%$ of mobile phase $\mathrm{A}$ and $55 \%$ of mobile phase $\mathrm{B}$ with flow rate of $0.35 \mathrm{~mL} \mathrm{~min}{ }^{-1}$; sample injection volume: $3 \mu \mathrm{L}$; total 
run time: $1.25 \mathrm{~min}$; column temperature: $35^{\circ} \mathrm{C}$. The data was processed using the software Mass Lynx ${ }^{\mathrm{TM}} 4.1$ and the results were expressed as $\mu \mathrm{g}$ per $\mathrm{g}$ of sample.

\section{Validation parameters}

The method validation parameters were determined according to the ICH guidelines. ${ }^{12}$ The figure of merit of the precision was obtained with six replicates and it was performed on the same day (intra-day). Analytical curves matched linearity, accuracy, linear range, limit of detection (LOD) and limit of quantification (LOQ). The LOD and LOQ were estimated basing on signal-to-noise ratios of 3 and 10 , respectively. All tests were under doped samples of each fruit.

\section{Results and Discussion}

\section{Analytical performance}

Figure 1 shows the chromatographic separation under optimized conditions of the equimolar mixture of the three anthocyanidins of interest. This result demonstrates that the separation of anthocyanidins can be achieved in a much smaller period of time (1.30 min) when compared to the conventional high pressure liquid chromatography (HPLC) runs, which are the most used for this purpose, with analytical runs of approximately 20 to $60 \mathrm{~min} .^{11,13-15}$

The spectra of fragmentation and product ion spectra obtained by direct infusion of analytical standards of anthocyanidins delphinidin, cyanidin and pelargonidin are shown in Supplementary Information. The mass/charge ratio $(m / z)$ values of these compounds, as well as the fragments resulting from electrospray ionization, were subsequently used in the quantification of these compounds in the samples. Due to the lack of elucidation regarding the compounds fragmentations, the Scheme 1 shows the proposed mechanisms of MS/MS collisional induced dissociation (CID) for pelargonidin $(\mathrm{m} / \mathrm{z}, 271)$, cyanidin $(\mathrm{m} / \mathrm{z}, 287)$ and delphinidin $(\mathrm{m} / \mathrm{z}, 303)$. The $\mathrm{m} / \mathrm{z}$ found in this analyzes corroborate with published results in samples containing anthocyanidins. ${ }^{16-18}$

The observed fragmentation using CID of all studied structures followed a pattern which leads to an elucidation of a general fragmentation mechanism. This kind of structure usually fragments into acylium ions. ${ }^{13}$ All the structures are rigid, and will need high amounts of energy to be cleaved, especially the closed shell $[\mathrm{M}+\mathrm{H}]^{+}$cation fragments obtained by hydrogen rearrangements and induced by charge dissociation. The first proposed dissociation mechanism pathway (I), present the ion of $m / z 69$ as common for all structures and it is proposed as a stable acylium ion. The second fragmentation pathway (II) leads to $\mathrm{H}_{2} \mathrm{O}$ loss and two consecutive $\mathrm{CO}$ losses. The third fragmentation route (III) leads to acylium ion formation followed by one CO loss. These three proposed fragmentation mechanisms cover almost all the observed ions in the CID spectra.

The $m / z$ ratio for the anthocyanidins of interest showed an error of approximately 0.05 Da for cyanidin, 0.11 Da for delphinidin and 0.14 Da for pelargonidin, when comparing them with the exact mass of the same molecules through the software ChemDraw Ultra 10.0 ${ }^{19}$ demonstrating the excellent resolution of the used spectrometer.
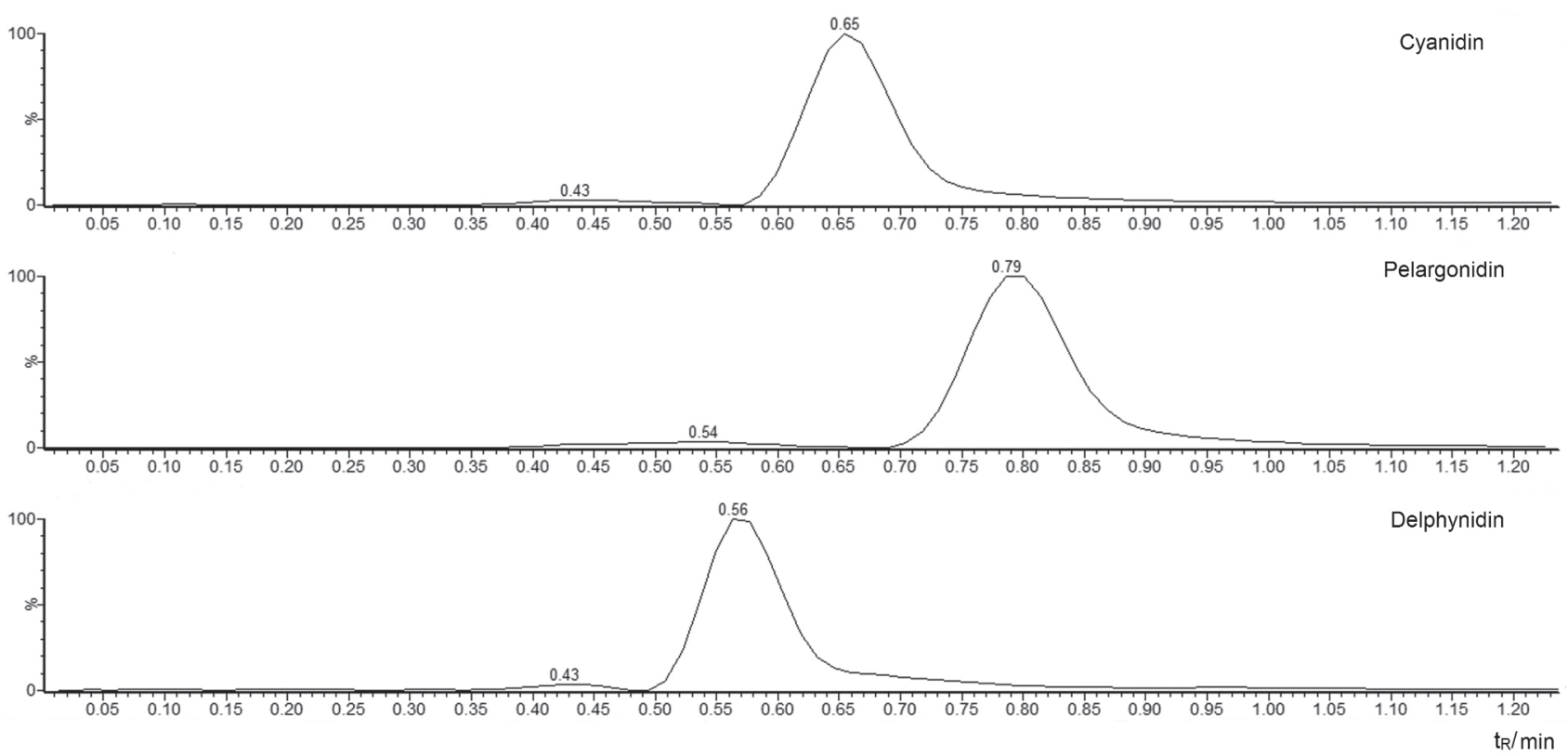

Figure 1. MRM chromatograms obtained from analytical standards of anthocyanidins. 


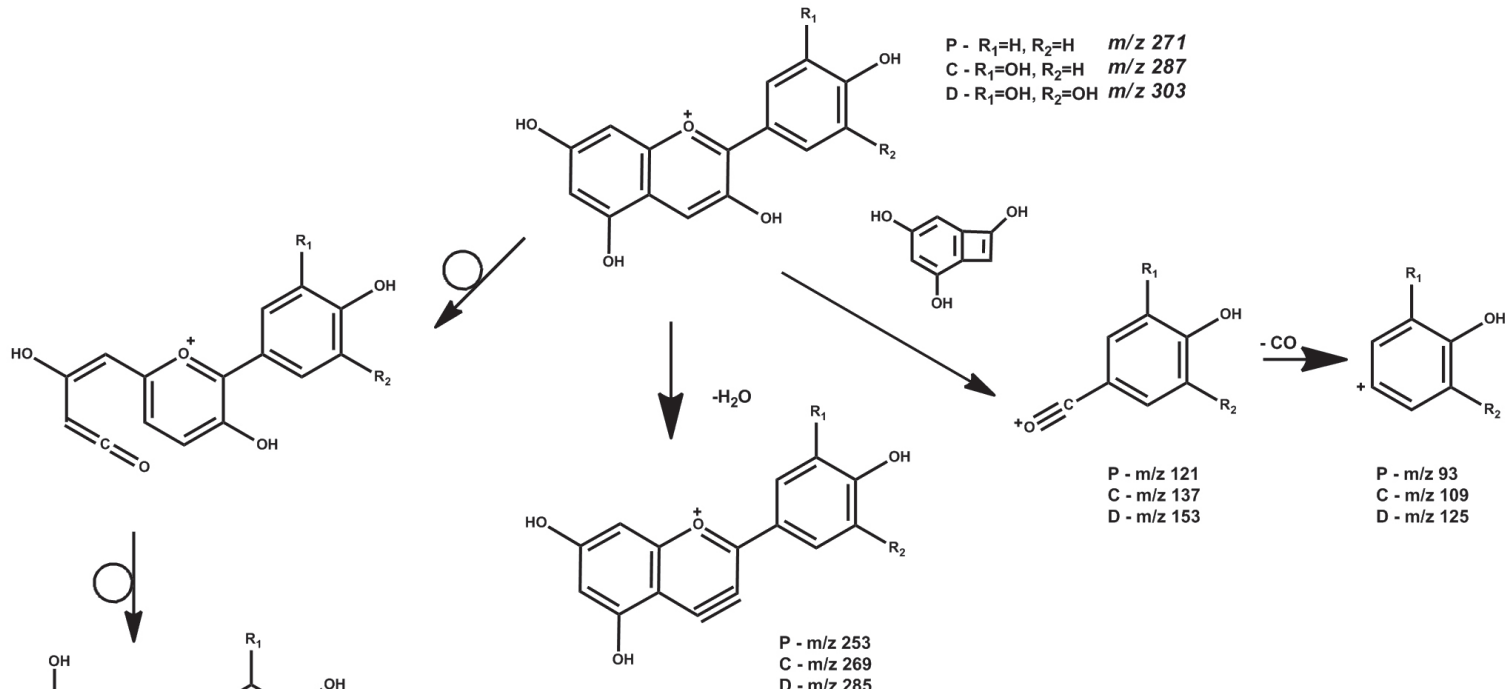<smiles>Cc1cc(C(=O)/C(O)=C\C=C\C(C)C=[IH])cc(C)c1O</smiles>

$-\mathrm{m} / \mathrm{z} 285$<smiles>CC(C)CC(C)C</smiles><smiles>N#CC=O</smiles><smiles>Oc1cccc(Br)c1</smiles>
$m / z 69$

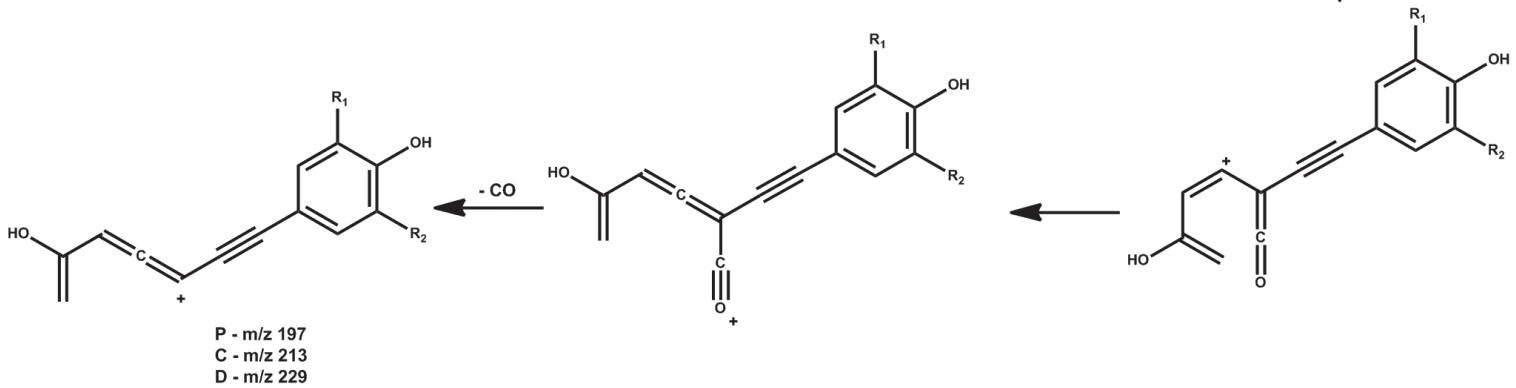

Scheme 1. Proposed fragmentation mechanism for protonated anthocyanin ions. (P) Pelargonidin; (C) cyanidin and (D) delphinidin.

With these first parameters defined, the results used for further validation and quantification of the proposed methodology are shown in Table 1.

For the three evaluated analytical standards, the following intervals were used in the construction of the calibration curves: $1 ; 5 ; 10 ; 25 ; 50 ; 100 ; 250 ; 500$ and $1000 \mathrm{ng}$. The calibration curves plotted by integrating the area of chromatographic peaks from each standard solution showed the following linear regressions: $y=11.1453 x-219.693$ with $\mathrm{r}^{2}=0.990912$ for cyanidin; $\mathrm{y}=12.6973 \mathrm{x}-1451.39$ with $\mathrm{r}^{2}=0.988477$ for pelargonidin and $\mathrm{y}=4.80831 \mathrm{x}-175.351$ with $r^{2}=0.993606$ for delphinidin.
For the effective validation of the proposed methodology, the figures of merit described in Table 2 were performed.

The results of Table 2 show all the samples created under doped of each fruit. The proposed technique presents excellent repeatability, as it presents a relative standard deviation of less than 5\% for all the investigated anthocyanins, high linearity (greater than 0.9 ) within the investigated range (1-1000 ng) and an accuracy value of less than $10 \%$. In addition, low limits of detection and quantification were achieved, important for the non-saturation of the column (extends its lifespan) while allowing both detection and quantification of 
Table 1. Selected ion transitions and optimized parameters for anthocyanidins analysis by UPLC-ESI-MS/MS

\begin{tabular}{lccc}
\hline Compound & Tr / min & Transition $(\mathrm{MRM})\left(\mathrm{H}^{+}\right)$ & Cone voltage / V \\
\hline Pelargonidin & 0.79 & $271.1>120.9^{\mathrm{a}}$ & 35 \\
& & $271.1>197.1^{\mathrm{b}}$ & 40 \\
Cyanidin & 0.65 & $287.1>137.1^{\mathrm{a}}$ & 70 \\
Delphinidin & & $287.1>121.0^{\mathrm{b}}$ & 45 \\
& 0.56 & $303.1>229.0^{\mathrm{a}}$ & 40 \\
\hline
\end{tabular}

${ }^{a}$ First transition used for quantitation; ${ }^{\mathrm{b}}$ second transition used for identification. UPLC-ESI-MS/MS: ultra performance liquid chromatography-electrospray ionization-mass spectrometry; MRM: multiple reaction monitoring.

Table 2. Figures of merits used for the validation of the proposed methodology (PM)

\begin{tabular}{|c|c|c|c|c|c|c|}
\hline Standard & $\begin{array}{c}\text { Precision / }\left(\mathrm{ng} \mathrm{mL} \mathrm{mL}^{-1}\right) \\
(\% \mathrm{RSD})\end{array}$ & Linearity $\left(r^{2}\right)$ & $\begin{array}{l}\text { Linear range / } \\
\left(\text { ng } \mathrm{mL}^{-1}\right)\end{array}$ & $\begin{array}{c}\left.\text { Accuracy / (ng mL } \mathrm{mL}^{-1}\right) \\
(\% \mathrm{RSD})\end{array}$ & $\mathrm{LOD} /\left(\mathrm{ng} \mathrm{mL} \mathrm{L}^{-1}\right)$ & $\begin{array}{c}\mathrm{LOQ} /\left(\mathrm{ng} \mathrm{mL} \mathrm{m}^{-1}\right) \\
(\% \mathrm{RSD})\end{array}$ \\
\hline Cyanidin & $100(4.36)$ & 0.990 & $1-1000$ & $100(4.45)$ & 5 & $10(4.6)$ \\
\hline Pelargonidin & $100(3.49)$ & 0.983 & $1-1000$ & $100(1.24)$ & 15 & $50(3.2)$ \\
\hline Delphinidin & $100(4.52)$ & 0.995 & $1-1000$ & $100(6.25)$ & 25 & $50(2.7)$ \\
\hline
\end{tabular}

RSD: relative standard deviation; LOD: limit of detection; LOQ: limit of quantification.

anthocyanidins at low concentrations in both fresh and processed samples such as fruit jellies and jams.

Usually, the extraction of these compounds is carried out in a medium acidified with hydrochloric acid $(\mathrm{HCl})$. However, in order to achieve an extraction based on the principles of green chemistry, it was proposed an extraction only with sample maceration. In addition, the commonly used analytical methodologies employ organic solvents, such as methanol and acetonitrile. Therefore, the development of an analytical methodology that uses water as mobile phase (just like the one which was carried out in this study) decreases the costs of the technique, besides not generating toxic residues.

\section{Determination of anthocyanidins in berries}

In order to verify the efficiency of the proposed methodology, the concentrations of anthocyanidins from berry samples were analyzed while comparing both extraction methodologies, and the results are shown in Table 3. The adequate concentrations were determined by applying the dilution factors required for the injected concentrations to be in the studied linear range. This is necessary in samples that contain high contents of analytes, because very high analyte concentrations may cause saturation of the chromatographic column. ${ }^{14}$

The information regarding the concentrations, the structure and the possible fragments coming from these flavonoids is important for the fields of analytical chemistry, food industry and food science, because anthocyanidins are natural pigments capable of being inserted into formulations in novel foods, and have different biological functions (antiinflammatory, anti-microbial, neuroprotective). ${ }^{15}$

Cyanidin is a potential protective agent against neurodegenerative disease ${ }^{20}$ by protecting the neuronal cells against oxidative processes, and this anthocyanin

Table 3. Anthocyanidins concentrations in six different berries

\begin{tabular}{lcccccc}
\hline \multirow{2}{*}{ Fruit } & \multicolumn{2}{c}{ Cyanidin $/\left(\mathrm{mg} \mathrm{g}^{-1}\right)^{\mathrm{a}}$} & \multicolumn{2}{c}{ Pelargonidin $/\left(\mathrm{mg} \mathrm{g}^{-1}\right)$} & \multicolumn{2}{c}{ Delphinidin / $\left(\mathrm{mg} \mathrm{g}^{-1}\right)$} \\
\cline { 2 - 7 } & $\mathrm{CM}$ & PM & CM & PM & CM & PM \\
\hline Morus nigra L. & $70.33 \pm 0.023$ & $74.53 \pm 0.025$ & $44.03^{\mathrm{b}} \pm 0.738$ & $5.65 \pm 0.121$ & $14.40 \pm 0.400$ & $12.90 \pm 0.688$ \\
Prunus avium L. & $30.10^{\mathrm{b}} \pm 0.015$ & $22.56 \pm 0.010$ & $45.20^{\mathrm{b}} \pm 0.605$ & $2.27 \pm 0.624$ & $14.53^{\mathrm{b}} \pm 0.354$ & $1.14 \pm 0.739$ \\
Rubus idaeus L. & $16.67 \pm 0.017$ & $22.42^{\mathrm{b}} \pm 0.015$ & $40.77^{\mathrm{b}} \pm 0.197$ & $3.07 \pm 0.160$ & $12.43^{\mathrm{b}} \pm 0.627$ & $1.40 \pm 0.569$ \\
Solanum americanum Mill. & $20.10^{\mathrm{b}} \pm 0.05$ & $6.69 \pm 0.03$ & $130.50^{\mathrm{b}} \pm 0.814$ & $41.24 \pm 0.955$ & $106.60 \pm 0.699$ & $292.67^{\mathrm{b}} \pm 0.927$ \\
Vaccinium myrtillus L. & $\mathrm{nd}$ & $4.29 \pm 0.013$ & $\mathrm{nd}$ & $75.42 \pm 0.165$ & $\mathrm{nd}$ & $11.40 \pm 0.248$ \\
Vitis vinifera L. & $15.03^{\mathrm{b}} \pm 0.026$ & $10.54 \pm 0.021$ & $47.53 \pm 1.280$ & $62.88^{\mathrm{b}} \pm 0.691$ & $27.77^{\mathrm{b}} \pm 0.515$ & $21.11 \pm 0.305$ \\
\hline
\end{tabular}

${ }^{a}$ per gram of fresh sample; ${ }^{b}$ significant differences between the same samples in different extraction method by $t$-test $(p<0.05)$. CM: conventional method; PM: proposed method. 
occurs with the highest frequency in edible fruits. ${ }^{15}$ Anthocyanidins are able to cross the blood-brain barrier, being promising in the prevention and treatment of brain cancer arising from alterations induced by epithelialmesenchymal transition. ${ }^{7}$ The antioxidant power of different anthocyanidins depends on the structure of each compound. The pelargonidin and delphinidin were the most potent antioxidants. ${ }^{2}$

Anthocyanidins in the free form have higher antioxidant activity than their glycosylated counterparts. ${ }^{21}$ Table 3 shows the concentration of anthocyanidins in different fruit samples.

Table 3 shows that cyanidin from Morus nigra L., Rubus idaeus L. and Vaccinium myrtillus L. was found in higher amounts in extracts obtained from the proposed method when compared with the conventional assay which uses $\mathrm{HCl}$. This may be justified since $\mathrm{HCl}$ is a strong acid and during the extraction process of anthocyanidins, it might react with part of the analytes through hydrolysis. ${ }^{22}$ The sample extracts of Prunus avium L., Solanum americanum Mill. and Vitis vinifera $\mathrm{L}$. showed higher concentrations of cyanidin in the conventional method, just like with pelargonidin and delphinidin. This can be justified by the fact that as anthocyanidins present higher concentrations in the glycosylated form, the correspondent mass values will be different from their non-glycosylated counterparts, and glycosylated anthocyanidins are not quantified by the proposed method in this work. ${ }^{18}$

The studied berries can be easily found worldwide, and can be consumed in natura, contributing for an adequate daily intake of bioactive nutrients. It is important to emphasize that berries have different concentrations of anthocyanidins, and it is important to vary between the types of berries, providing the body with different types of anthocyanidins and thus contributing to the maintenance of a healthy organism.

\section{Conclusions}

The developed method enables the extraction of the compounds of interest without using toxic solvents, in addition to the detection and quantification of analytes on the scale of nanograms in just $1.30 \mathrm{~min}$. It is also relevant to mention that this is the first time that concentrations of anthocyanidins present in fruits of Solanum americanum Mill. were reported. When carrying out the quantification of anthocyanidins, a prior study must be done in order to confirm if the samples have anthocyanidins in free or glycosylated form. The non-glycosylated standards must be purchased in order to be able to quantify analytes in free form, otherwise, the quantification will not be correct. When carrying out the extraction with the conventional assay which uses $\mathrm{HCl}$, the anthocyanidins may be hydrolyzed (if they are in free form), thereby reducing their concentration to be extracted.

\section{Supplementary Information}

Supplementary data (mass spectra) are available free of charge at http://jbcs.sbq.org.br as PDF file.

\section{Acknowledgments}

This work was supported by the Coordenação de Aperfeiçoamento de Pessoal de Nivel Superior (CAPES).

\section{References}

1. Bourgaud, F.; Gravot, A.; Milesi, S.; Gontier, E.; Plant Sci. 2001, 161, 839.

2. Dimitrić Marković, J. M.; Pejin, B.; Milenković, D.; Amić, D.; Begović, N.; Mojović, M.; Marković, Z. S.; Food Chem. 2017, 218,440 .

3. Winkel-Shirley, B.; Plant Physiol. 2001, 26, 485.

4. Liu, R. H.; J. Nutr. 2004, 134, 3479S.

5. Yıldırım, S.; Kadığlu, A.; Sağlam, A.; Yaşar, A.; Sellitepe, H. E.; J. Sep. Sci. 2016, 39, 3927.

6. Kubota, M.; Ishikawa, C.; Sugiyama, Y.; Fukumoto, S.; Miyagi, T.; Kumazawa, S.; J. Food Compos. Anal. 2012, 28, 179.

7. Ouanouki, A.; Lamy, S.; Annabi, B.; Mol. Carcinog. 2017, 56, 1088.

8. Mazza, G.; Cacace, J. E.; Kay, C. D.; J. AOAC Int. 2004, 87, 129.

9. Spiro, T. G.; Stigliani, W. M.; Yamamoto, S. M.; Química Ambiental; Pearson Prentice-Hall: São Paulo, 2009.

10. Silva, F. M.; Lacerda, P. S. B.; Junior, J. J.; Quim. Nova 2005, 18, 103.

11. Babou, L.; Hadidi, L.; Grosso, C.; Zaidi, F.; Valentão, P.; Andrade, P. B.; Eur. Food Res. Technol. 2016, 242, 1447.

12. International Conference on Harmonization (ICH); Validation of Analytical Procedures: Text and Methodology Q2 (R1); ICH: Geneva, 2005.

13. Meurer, E. C.; Sparrapan, R.; Tomazela, D. M.; Eberlin, M. N.; Augusti, R.; J. Am. Soc. Mass Spectrom. 2005, 16, 1602.

14. Morrison, R. D.; Dolan, J. W.; LCGC North Am. 2005, 23, 566.

15. Olivas-Aguirre, F.; Rodrigo-García, J.; Martínez-Ruiz, N.; Cárdenas-Robles, A.; Mendoza-Díaz, S.; Álvarez-Parrilla, E.; González-Aguilar, G.; de la Rosa, L.; Ramos-Jiménez, A.; Wall-Medrano, A.; Molecules 2016, 21, 1264.

16. Guimarães, W.; Alves, M. I. R.; Filho, N. R. A.; Quim. Nova 2012, 35, 1673. 
17. Zhang, Z.; Kou, X.; Fugal, K.; McLaughlin, J.; J. Agric. Food Chem. 2004, 52, 688.

18. Abrankó, L.; Szilvássy, B.; J. Mass Spectrom. 2015, 50, 71.

19. Mills, N.; J. Am. Chem. Soc. 2006, 128, 13649.

20. Thummayot, S.; Tocharus, C.; Suksamrarn, A.; Tocharus, J.; Neurochem. Int. 2016, 101, 15.

21. Kähkönen, M. P.; Heinämäki, J.; Ollilainen, V.; Heinonen, M.; J. Sci. Food Agric. 2003, 83, 1403.
22. Yousef, G. G.; Brown, A. F.; Funakoshi, Y.; Mbeunkui, F.; Grace, M. H.; Ballington, J. R.; Loraine, A.; Lila, M. A.; J. Agric. Food Chem. 2013, 61, 4806.

Submitted: February 17, 2017

Published online: June 19, 2017 